\title{
Lessons of the month 3: Gone but not forgotten - Osler - a reminder of the syndrome not bearing his name
}

\author{
Authors: Amit KJ Mandal, ${ }^{A}$ Bashir Mohamad ${ }^{B}$ and Constantinos G Missouris ${ }^{\mathrm{C}}$
}

Streptococcus pneumoniae is the most frequently implicated microbial agent in community acquired bacterial pneumonia and meningitis. It is also responsible for between 1 and $3 \%$ of cases of native valve infective endocarditis, with mortality rates up to $60 \%$. Osler first described the association between pneumococcal pneumonia, endocarditis, and meningitis secondary to bacteria that he described as 'micrococci', subsequently elucidated to be $S$ pneumoniae by Robert Austrian, and the syndrome bears his name. We report a case of fulminant pneumococcal native aortic valve endocarditis and perforation in a young male patient with chronic alcoholism and splenectomy who exhibited poor compliance to pneumococcal prophylaxis.

KEYWORDS: Osler, Streptococcus pneumoniae, endocarditis, splenectomy

\section{Case presentation}

A 39-year-old independent man was admitted to our hospital after a witnessed self-limiting grand mal seizure. He had been unwell for a week with fever and cough productive of rusty sputum.

Past medical history was significant for alcohol dependence, chronic pancreatitis, type 2 diabetes mellitus requiring insulin, and splenectomy 4 years previously following traumatic injury. He was non-compliant with pneumococcal antibiotic prophylaxis and immunisation status was unknown. His usual alcohol consumption was six-seven pints of strong lager per day (>100 units per week).

On examination there was low-grade fever $\left(37.6^{\circ} \mathrm{C}\right)$, tachypnoea and signs of consolidation in the right lung. There was no neck stiffness or rash and cardiovascular examination was normal. Chest radiography confirmed dense consolidation in the right upper lobe (Fig 1).

Laboratory investigations were notable for haemoglobin of $117 \mathrm{~g} / \mathrm{L}$, white cell count of $14.71 \times 10^{9} / \mathrm{L}$ (neutrophils $13.29 \mathrm{x}$ $10^{9} / \mathrm{L}$ ), platelets of 171 , and a C-reactive protein at $281 \mathrm{mg} / \mathrm{L}$.

Authors: ${ }^{\text {A }}$ consultant, Wrexham Park Hospital, Slough, UK; ${ }^{B}$ consultant, Wrexham Park Hospital, Slough, UK; ' Consultant, Wrexham Park Hospital, Slough, UK and professor, University of Cyprus Medical School, Nicosia, Cyprus

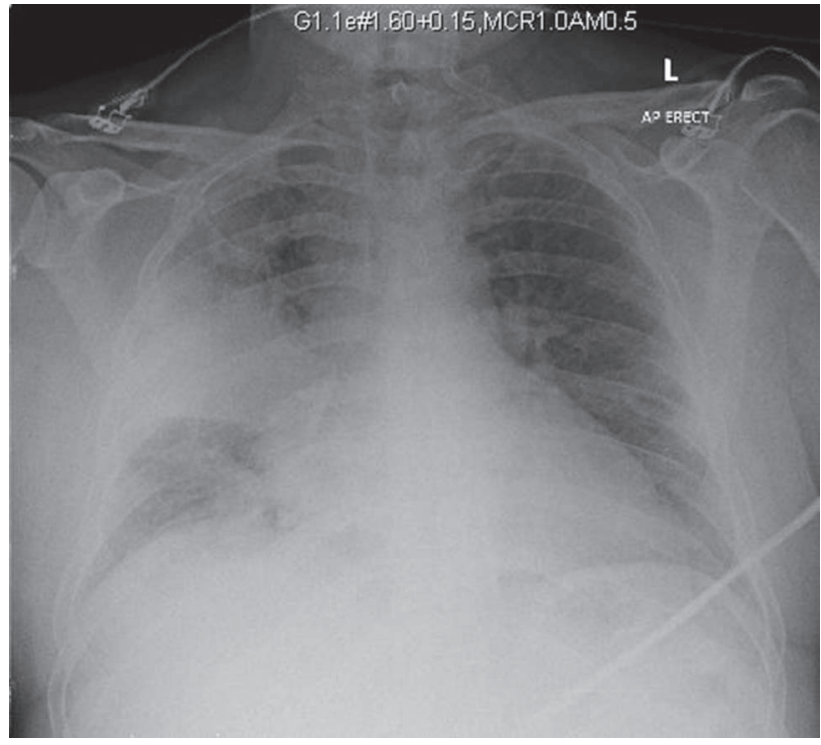

Fig 1. Admission chest radiography demonstrating dense consolidation in the right upper lobe.

Markers of renal and hepatic function were normal. Urine dip showed red blood cells. The patient was commenced on intravenous co-amoxiclav and gentamicin in addition to chlordiazepoxide and B vitamin complex for alcohol withdrawal.

Within 24 hours of admission there was progressive type 1 respiratory failure and reduced level of consciousness requiring intubation and ventilation. Two sets of blood cultures were positive for Streptococcus pneumoniae and the antimicrobial regimen was accordingly changed to a combination of ceftriaxone, rifampicin and gentamicin. Despite this there was ongoing disseminated bacteraemia with increasing oxygen requirements, persistent fever, rising inflammatory markers, and a widening pulse pressure. On day 12 , transoesophageal echocardiography confirmed the presence of native aortic valve endocarditis with perforation of the left coronary cusp resulting in severe aortic regurgitation and a globally dilated left ventricle with poor systolic function (Fig 2).

The patient remained neurologically unstable and magnetic resonance imaging (MRI) of the brain showed multiple foci of restricted diffusion in the left cerebellar, parietal and subcortical 


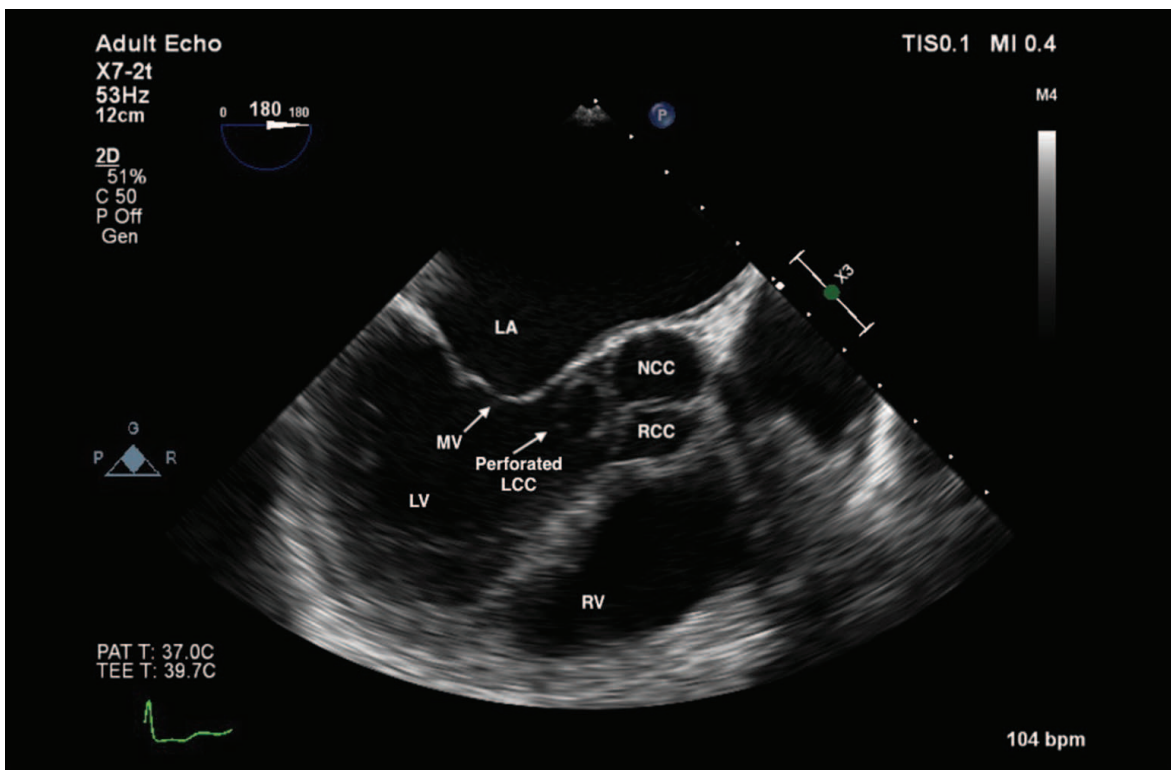

Fig 2. Transoesophageal echocardiography demonstrating aortic valve endocarditis with perforation of the left coronary cusp. The other two cusps were seemingly unaffected. There was severe aortic regurgitation and the left ventricle was dilated with poor systolic function globally. $\mathrm{LA}=$ left atrium; $\mathrm{LCC}=$ left coronary cusp; $\mathrm{LV}=$ left ventricle; $\mathrm{MV}=$ mitral valve; $\mathrm{NCC}=$ non-coronary cusp; $\mathrm{RCC}=$ right coronary cusp; $\mathrm{RV}=$ right ventricle.

areas, consistent with septic emboli. Electroencephalography (EEG) revealed a diffuse slow wave pattern, composed of undulating symmetrical delta wave activity, in keeping with underlying severe encephalopathy. However, at this stage, no bacteria were detected by direct microscopy of cerebrospinal fluid (CSF), and CSF cultures were unyielding.

Urgent aortic valve replacement with a $25 \mathrm{~mm}$ Edwards Inspiris Resilia pericardial bioprosthesis was performed with aortocoronary vein bypass to the mid left anterior descending coronary artery. At operation the heart was found to be dilated and sluggish. There was free aortic regurgitation with active infection and perforation of the left coronary cusp without evidence of aortic root abscess. The patient made a slow and uneventful recovery and was discharged from hospital 73 days after admission, having completed 6 weeks of intravenous rifampicin and ceftriaxone antimicrobial therapy. Transthoracic echocardiography performed prior to discharge confirmed normalisation of the left ventricular systolic function with a wellfunctioning aortic valve prosthesis.

\section{Discussion}

Among patients with a history of alcohol abuse and splenectomy, who present with $S$ pneumoniae sepsis failing to improve with appropriate antibiotic therapy, a high index of suspicion should be carried for concomitant endocarditis. As far as we are aware, this is the first report of fulminant pneumococcal native aortic valve endocarditis and perforation in a patient with splenectomy who exhibited poor compliance to pneumococcal prophylaxis.

The association between pneumonia, meningitis and endocarditis was first described by William Osler in $1881 .{ }^{1}$ He referred to ulcerative endocarditis as "one of the most formidable of cardiac affections, characterised by a peculiar morbid process on the valves, blood contaminations, constitutional symptoms and usually associated with multiple emboli.' Osler appreciated the infectious nature of endocarditis and even observed the presence of 'micrococci' in histological specimens at post-mortem examination.
He noted that a number of his patients also had pneumonia and assumed that endocarditis developed after lung infection. In 1957 Robert Austrian further classified these micrococci as $S$ pneumoniae, hence the eponym, Austrian syndrome. ${ }^{2}$

The incidence of $S$ pneumoniae endocarditis stands at $3 \%$ of all cases of endocarditis compared to $10-15 \%$ in the pre-antibiotic era. Nowadays, Austrian syndrome, a clinical rarity, sparsely reported in the literature, has an incidence of approximately $1 \%$ of all cases of endocarditis. It is a lethal entity with mortality rates up to $60 \%$. There is a strong association with alcoholism and chronic disease states. ${ }^{3-5}$ The reason is unclear, but we postulate that it may be related to general immunosuppression and malnutrition.

Interestingly, underlying valvular heart disease does not appear to lend itself as a strong risk factor. $S$ pneumoniae appears to have an affinity to the native aortic valve and leads to acute and aggressive valve destruction that may also involve the aortic root leading to severe aortic insufficiency, and survival is contingent upon immediate valve replacement. Less than one-third of cases have a subacute evolution involving the mitral valve and this affords a comparatively better outcome with a longer antimicrobial sterilisation window prior to valve replacement.

\section{Conclusion}

Emphasis is therefore laid on the importance of screening for endocarditis among patients with $S$ pneumoniae infections, particularly in the context of chronic alcoholism (even with normal synthetic function as in our patient) or splenectomy. We should remember Osler's mantra, that 'medicine is a science of uncertainty and an art of probability', and accordingly, exercise a high index of suspicion for native aortic valve endocarditis in patients whose symptoms and signs can be explained by meningitis and pneumonia, but which fail to respond to appropriate antimicrobial therapy based on blood culture susceptibility. Only expeditious medical therapy and early aortic 
valve replacement is likely to reduce morbidity and mortality in this unusually brutal entity.

We also propose that this syndrome should be renamed as Osler's syndrome with Osler's tetrad, in acknowledgement that Sir William Osler was the first to describe the triad of pneumonia, meningitis and endocarditis with presence of 'micrococci' in affected tissues and blood.

\section{References}

1 Osler W. Infectious (so called ulcerative) endocarditis. The Archives of Medicine, New York 1881;5:44-68.

2 Austrian R. Pneumococcal endocarditis, meningitis, and rupture of the aortic valve. AMA Arch Intern Med 1957:99:539-44.
3 Gonzalez-Juanatey C, Testa A, Mayo ] et al. Austrian syndrome and review of two new cases and literature review. Int J Cardiol 2006:108:273-5.

4 Gransden WR, Eykyn S], Phillips I. Pneumococcal bacteria emia: 325 episodes diagnosed at St Thomas's Hospital. BMJ (Clin Res Ed) 1985:290:505-8.

5 Aronin SI, Mukherjee SK, West IC et al. Review of pneumococcal endocarditis in adults in the penicillin era. Clin Infect Dis 1998;26:165-71.

Address for correspondence: Prof Constantinos G Missouris, Departments of Medicine and Cardiology, Wexham Park Hospital, Frimley Health NHS Foundation Trust, Wexham Street, Slough SL2 4HL, UK.

Email: dinos.missouris@nhs.net

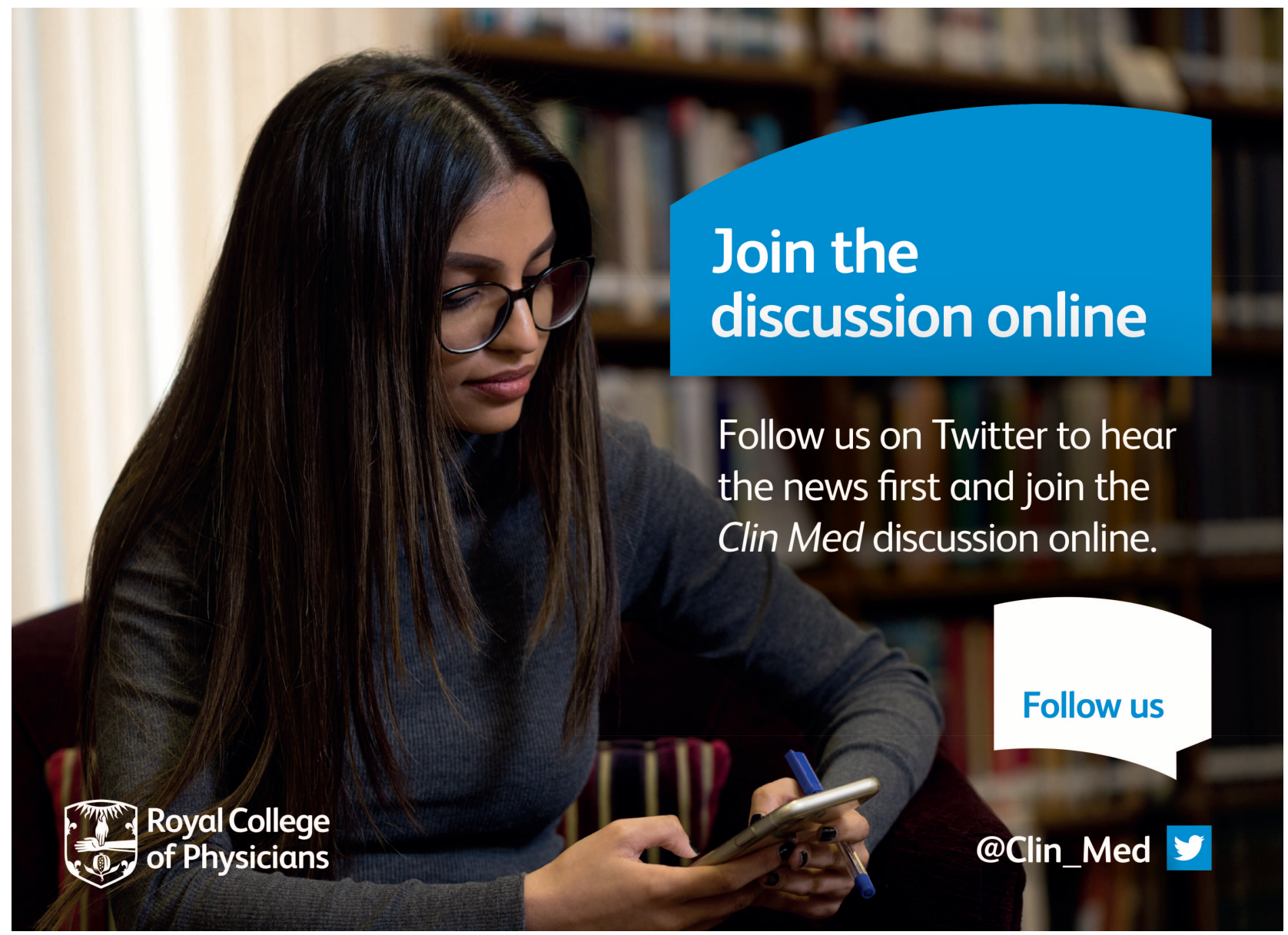

